\title{
System-wide molecular dynamics of endothelial dysfunction in Gram-negative sepsis
}

Xavier Gallart-Palau ${ }^{1,2,3,4,5,6}$, Aida Serra ${ }^{5,6^{*}}$ and Siu Kwan Sze

\begin{abstract}
Background: Inflammation affecting whole organism vascular networks plays a central role in the progression and establishment of several human diseases, including Gram-negative sepsis. Although the molecular mechanisms that control inflammation of specific vascular beds have been partially defined, knowledge lacks on the impact of these on the molecular dynamics of whole organism vascular beds. In this study, we have generated an in vivo model by coupling administration of lipopolysaccharide with stable isotope labeling in mammals to mimic vascular beds inflammation in Gram-negative sepsis and to evaluate its effects on the proteome molecular dynamics. Proteome molecular dynamics of individual vascular layers (glycocalyx (GC), endothelial cells (EC), and smooth muscle cells $(S M C))$ were then evaluated by coupling differential systemic decellularization in vivo with unbiased systems biology proteomics.

Results: Our data confirmed the presence of sepsis-induced disruption of the glycocalyx, and we show for the first time the downregulation of essential molecular maintenance processes in endothelial cells affecting this apical vascular coating. Similarly, a novel catabolic phenotype was identified in the newly synthesized EC proteomes that involved the impairment of protein synthesis, which affected multiple cellular mechanisms, including oxidative stress, the immune system, and exacerbated EC-specific protein turnover. In addition, several endogenous molecular protective mechanisms involving the synthesis of novel antithrombotic and anti-inflammatory proteins were also identified as active in EC. The molecular dynamics of smooth muscle cells in whole organism vascular beds revealed similar patterns of impairment as those identified in EC, although this was observed to a lesser extent. Furthermore, the dynamics of protein posttranslational modifications showed disease-specific phosphorylation sites in the EC proteomes.
\end{abstract}

Conclusions: Together, the novel findings reported here provide a broader picture of the molecular dynamics that take place in whole organism vascular beds in Gram-negative sepsis inflammation. Similarly, the obtained data can pave the way for future therapeutic strategies aimed at intervening in specific protein synthesis mechanisms of the vascular unit during acute inflammatory processes.

Keywords: Vascular beds, Lipopolysaccharide, Endothelial dysfunction, Inflammation, Infection, DISDIVO

\footnotetext{
*Correspondence: aida.serra@imdea.org; sksze@ntu.edu.sg

${ }^{5}$ IMDEA Food \& Health Sciences Research Institute, +Pec Proteomics, Campus

of International Excellence UAM+CSIC, Old Cantoblanco Hospital, 8 Crta.

Canto Blanco, 28049 Madrid, Spain

${ }^{1}$ School of Biological Sciences, Nanyang Technological University, 60

Nanyang Drive, Singapore 637551, Singapore

Full list of author information is available at the end of the article
}

(C) The Author(s). 2020 Open Access This article is licensed under a Creative Commons Attribution 4.0 International License, which permits use, sharing, adaptation, distribution and reproduction in any medium or format, as long as you give appropriate credit to the original author(s) and the source, provide a link to the Creative Commons licence, and indicate if changes were made. The images or other third party material in this article are included in the article's Creative Commons licence, unless indicated otherwise in a credit line to the material. If material is not included in the article's Creative Commons licence and your intended use is not permitted by statutory regulation or exceeds the permitted use, you will need to obtain permission directly from the copyright holder. To view a copy of this licence, visit http://creativecommons.org/licenses/by/4.0/ The Creative Commons Public Domain Dedication waiver (http://creativecommons.org/publicdomain/zero/1.0/) applies to the data made available in this article, unless otherwise stated in a credit line to the data. 


\section{Background}

Homeostasis in all systems of the human body depends, to a large extent, on the molecular and structural integrity of the cardiovascular system (CVS). This intricate and supportive system provides adaptive metabolic supplementation of nutrients, molecular messengers, and oxygen to cells, while it eliminates unwanted residues and sustains immunity $[1,2]$. The CVS is formed by a vast network of vessels that vary in length, diameter, and function, with endothelial cell (EC) and glycocalyx (GC) layers at the inner areas of vasculature beds [2]. Additionally, arteries and veins are formed by vascular smooth muscle cells (SMC), a population of innervated cells with the ability to regulate vascular tone in conjunction with EC [3, 4].

Dysfunction of the endothelium is associated with the appearance and progression of the most severe human diseases, including sepsis, diabetes, stroke, dementia, and cancer [5-10]. Although these diseases are characterized by specific alterations of the endothelium, some of which have yet to be fully elucidated, inflammation has been defined as a core pathological mechanism affecting vascular beds in all these pathologies [11, 12]. Similarly, inflammation has been found to be a core mechanism of microvasculature disruption preceding organ dysfunction in sepsis [13]. Of note, recent epidemiological compilations indicate that the burden of sepsis exceeds that of cancer globally, and it has become the second-ranked global cause of death behind only cardiovascular diseases [14, 15].

Lipopolysaccharide (LPS), also known as endotoxin, is a bacterial molecule centrally implicated in the pathogenesis of severe sepsis and septic shock [16]. This circulating toxin has the ability to indicate the occurrence of sepsis in blood while activating the systemic release of a myriad of pro-inflammatory molecules [17]. These proinflammatory factors are known to disrupt vascular beds by promoting the apoptosis of $\mathrm{EC}$, which in turn leads to edema formation and organ failure $[18,19]$. Although LPS-induced disruption of the endothelial barrier has been thoroughly described in specific vascular beds, such as the lungs and liver, the comprehension of the molecular events that precede vascular bed disruption in severe sepsis remains poor. Similarly, further light needs to be shed on the effects of LPS throughout the whole organismal network of capillary beds. According to the recent literature review performed by Libert et al. [20], LPS has been used in one third of the most relevant studies that involve the use of animal models in sepsis. However, it has been admitted that sepsis is caused by a highly complex pathophysiology that cannot be fully mimicked using LPS in rodents [20]. The use of LPS, thus, should be limited to mimicking specific relevant clinical features of sepsis in a robust, quick, precise, and highly replicable manner, especially the severe inflammatory response that affects the endothelium in this disease and causes the appearance of fever, leukocytosis, and cytokine release, among other features [20].

Alterations in protein synthesis can be considered among the earliest molecular events of disease progression [21, 22]. Bacterial pathogenesis has recently been associated with alterations in protein synthesis in platelets and gut epithelial cells [23, 24], although little is still known about how the cell renewal mechanism becomes impaired in the whole organism vascular bed layers. Novel systems biology methods coupled to the study of whole organism vascular beds hold promise for advancing the understanding of the effects of sepsis on protein synthesis in vascular and capillary beds. Thus, in this study, we combined for the first time stable isotope labeling of mammals (SILAM) $[25,26]$ with differential systemic decellularization in vivo (DISDIVO) [27] to characterize the molecular dynamics of whole organism vascular beds in Gram-negative sepsis. SILAM, as initially reported by Yates, J.R. III, and colleagues [28] is based on the depletion of light proteins (proteins without isotope-labeled Lys) in the dietary protein source of the animal by substitution of these with heavy proteins (proteins with isotope-labeled Lys). Thus, all newly synthesized proteomes in the animal incorporate isotopelabeled Lys, which can in turn be easily identified/quantified by mass spectrometry [28]. Similarly, DISDIVO allows systemic decellularization of independent vascular layers and analysis of the vascular layers proteomes by systems biology [27]. Although optimization of the DISD IVO method demonstrated that the conditions used for the obtention of each independent vascular layer are the most experimentally appropriate, the possibility that cross-contamination between vascular mantles occurs in DISDIVO cannot be discarded. However, based on the extensive imaging and bioinformatics analysis performed on data generated by this method, the systematic approach of DISDIVO involving the entire vascular system seems able to compensate for these potential peculiarities. Our novel findings indicate that dramatic inhibition of protein synthesis and partial protein synthesis shift occur in whole organism vascular bed layers together with abnormal protein turnover in EC. Thus, the findings uncovered here highlight specific interference by endotoxemia in the regular molecular dynamics of the whole organism endothelium during acute inflammatory processes.

\section{Results}

Use of SILAM-DISDIVO for the study of whole organism vascular beds in Gram-negative sepsis

To study the effect(s) of the endotoxin LPS on the proteome dynamics of whole organism vascular beds during 
sepsis, we made use of a SILAM model generated by replacing dietary Lys with the stable isotope Lys(6). Validation of DISDIVO fractions in whole SILAM mouse vascular beds (GC, EC, and SMC) revealed the proper incorporation of Lys(6) into newly synthesized proteins, as shown in Fig. 1. Lys(6) was incorporated on average in a total of $350 \pm 112$ newly synthesized proteins in vascular bed proteomes, which represented $31 \%$ of the total proteome in the GC in control mice, $29 \%$ of the total proteome in EC, and $34 \%$ of the total proteome in SMC in control mice. In addition, in Gram-negative sepsis, newly synthesized proteins represented only $16 \%$ of the total proteome in the GC, $18 \%$ of the total proteome in EC, and 16\% of the total proteome in SMC (Fig. 1a,

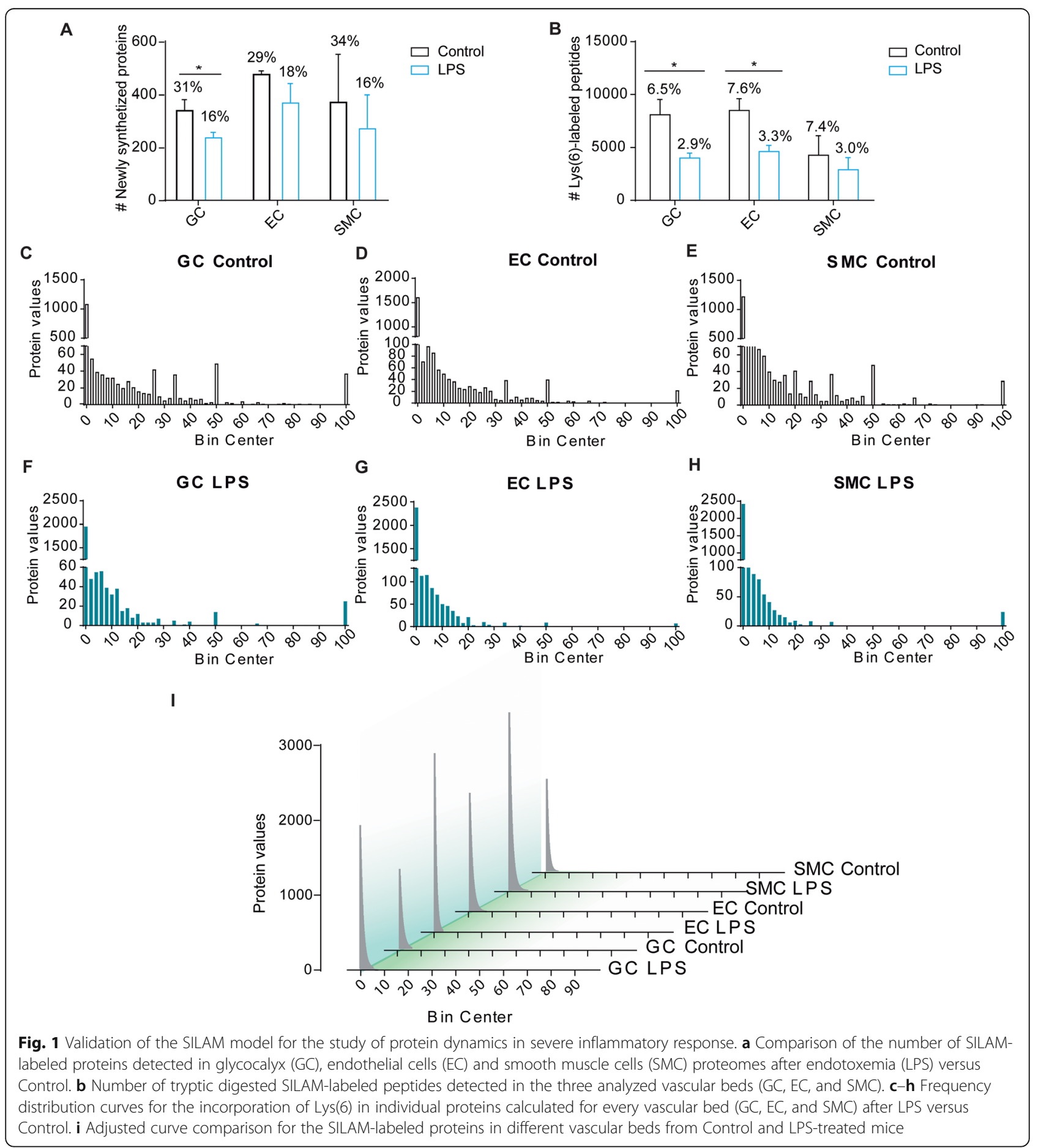


Additional File 1: Dataset 1 and Additional File 2: Dataset 2). Finally, the validated SILAM model also revealed the efficient averaged labeling of $\operatorname{Lys}(6)$ in a total of $5.1 \pm 2.3 \%$ of all analyzed peptidomes (Fig. 1b).

\section{Molecular dynamics of whole organism vascular beds during Gram-negative sepsis}

Detailed analysis of the molecular dynamics occurring in whole organism vascular beds during Gram-negative sepsis indicated that LPS challenge triggers a significant, rapid, and severe reduction in the molecular maintenance of the GC. Thus, a significant decrease in the total number of Lys(6)-labeled proteins detected in this vascular coating layer was observed, as shown in Fig. 1a. Similarly, a slight reduction in the total number of newly synthesized proteins was observed in the EC and SMC vascular layers in Gram-negative sepsis, although these differences did not reach statistical significance (Fig. 1a).

The frequency of Lys(6) incorporation in newly synthesized proteins in Gram-negative sepsis was also investigated. The obtained data showed narrowing of the cumulative frequency curves of the proteomes in LPStreated vascular beds (Fig. 1c-i). This result clearly indicates the reduced incorporation of Lys(6) into individual proteins in all of the analyzed vascular beds during Gram-negative sepsis (Fig. 1c-i). To further scrutinize this finding, the LPS/Control ratios for newly synthesized proteins $\left(\mathrm{NSP}_{\mathrm{LPS} / \mathrm{Control}}\right)$ and non-newly synthesized proteins $\left(\mathrm{N}-\mathrm{NSP}_{\mathrm{LPS} / \mathrm{Control}}\right)$ were calculated for all $\mathrm{EC}$ and SMC proteomes. In EC, all endotoxinmodulated proteins $(p \leq 0.05)$ showed a dramatically downregulated $\mathrm{NSP}_{\mathrm{LPS} / \text { Control }}$ ratio (Fig. 2a, b), except for hemopexin precursor protein $(\mathrm{Hpx})$, which was highly upregulated in LPS-treated EC (Fig. 2a, b). Our data also demonstrated that SMC proteomes were less affected by the effects of endotoxin-induced sepsis on protein synthesis compared to EC proteomes, as can be observed through the reduced clustering of modulated proteins in these specific vascular beds (Fig. 2c, d). The $\mathrm{NSP}_{\text {LPS/Control }}$ ratio was similarly downregulated in SMC for all endotoxin-modulated proteins, as shown in Fig. 2c, except for the protein serine protease inhibitor (Serpina3n).

In a related vein, we found that endotoxemia triggered new synthesis of a specific subset of proteins in EC and SMC during Gram-negative sepsis, as shown in Fig. 2b, d. Proteins exclusively synthesized in LPS-challenged EC vascular beds included haptoglobin $(\mathrm{Hp})$ and serum amyloid A1 (Saa1) (Fig. 2b), whereas S100 calciumbinding protein A9 (S100a9), galectin-1 (Lgals1), and a small cluster of histones were exclusively synthesized in challenged SMC, as shown in Fig. 2d.

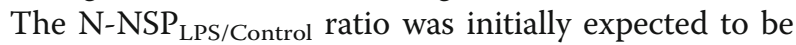
close to 1; however, we found that this ratio was strikingly modulated in a wide range of proteins in EC during Gram-negative sepsis, as shown in Fig. 2a. Further investigation of this subset of EC-specific modulated proteins indicated the increased abundance (or exclusive presence) of nontryptic peptides derived from these proteins in LPS-challenged animals, which, based on previous findings [29], clearly indicated the occurrence of active protein turnover in EC during Gramnegative sepsis (Fig. 2e).

Proteins with high turnover rates included vascular cell adhesion molecule 1 (VCAM1), the inflammationrelated protein serum amyloid A (Saa2), and cytochrome c oxidase subunit $5 \mathrm{~B}$ (Cox $5 \mathrm{~b})$. On the other hand, the protein turnover rates of alpha-2-macroglobulin $(\alpha 2 \mathrm{M})$, $\beta 2$ microglobulin $(\beta 2 \mathrm{M})$, and orosomucoid 2 (Orm2) were found to be significantly downregulated in LPSchallenged EC vascular beds (Fig. 2e).

\section{Functional characterization of GC molecular dynamics}

Although the GC in whole body vascular beds functions as a vasculature mantle that lacks protein synthesis ability, the GC is predictably one of the most variably affected vascular layers during Gram-negative sepsis. Thus, to further characterize any potential abnormal incorporation of newly synthesized proteins into the GC, we performed an in-depth characterization of GC Lys(6)-labeled proteomes. These analyses, as expected, revealed a reduction in proper GC molecular maintenance, which was linked to a significantly decreased abundance of important cellular mechanism-related proteins (Fig. 3a, b). These included lipid transport apolipoproteins (Fig. 3b-I), immune-related proteins (Fig. 3b-II), including fetuin-B (fetub) and integrin alpha-IIb (Itga2b), and several component proteins. Other affected GC cellular mechanism-related proteins included pro-atherosclerotic proteins, oxidative stressrelated proteins, coagulation cascade proteins, and, of note, abnormally incorporated, newly synthesized structural/cell signal transduction proteins, among others (Fig. 3a, b-III to VII).

\section{Functional characterization of EC and SMC molecular dynamics}

To investigate the most dramatically affected cellular mechanisms in EC and SMC in endotoxin-induced sepsis, we analyzed the Lys(6)-labeled EC and SMC proteomes from LPS-challenged and control mice by systems biology, as shown in Fig. 4a, b-I to VIII. The initiation of EC dysfunction was clearly induced by the downregulation of the synthesis of multiple key proteins, including clusterin $(\mathrm{Clu})$, glutathione $\mathrm{S}$-transferase $\mathrm{Mu} 1$ (Gstm1), and selenoprotein P (Sepp1), among others (Fig. 4b-VI). Similarly, the synthesis of endothelial oxidation- and endothelial structure-related proteins was also negatively modulated (Fig. 4b-III and VII). Other cellular 


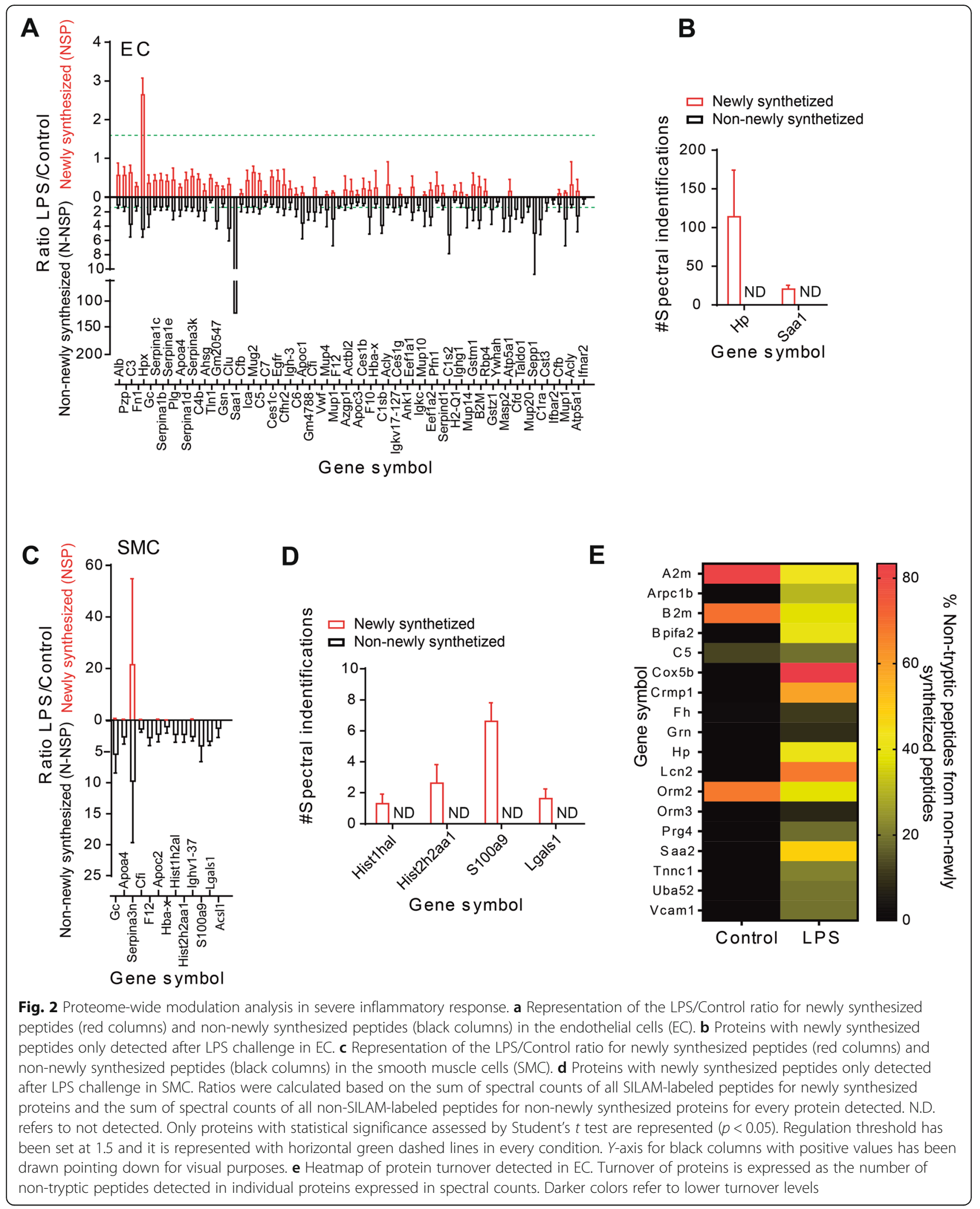

mechanisms significantly affected by the endotoxemic challenge included coagulation, which experienced the significant downregulation of the synthesis of coagulation factor X (F10) and heparin cofactor 2 protein (Serpind1) (Fig. 4b-V), and metabolism-related proteins through the downregulation of carboxylesterase $1 \mathrm{C}$ 
A

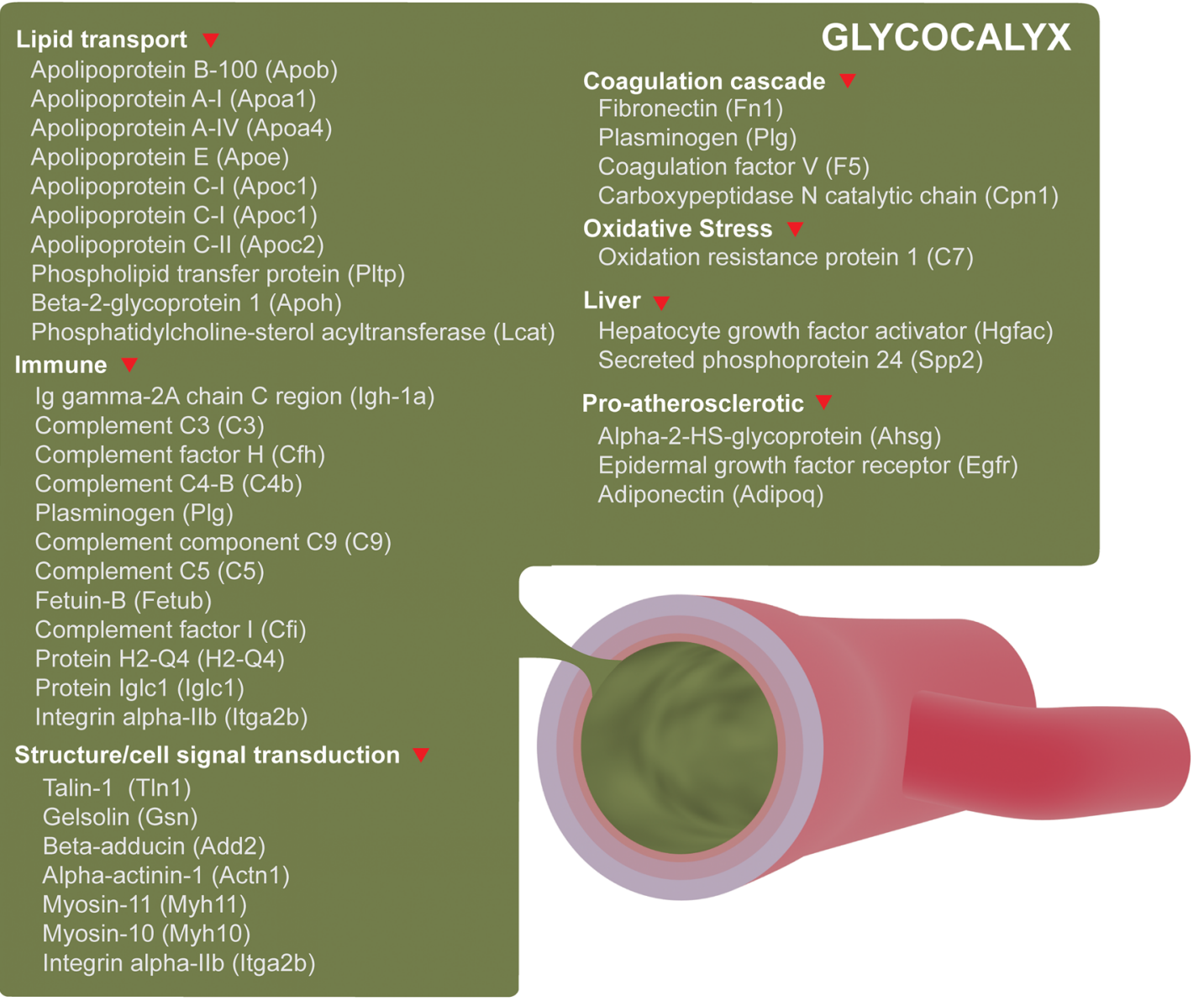

B
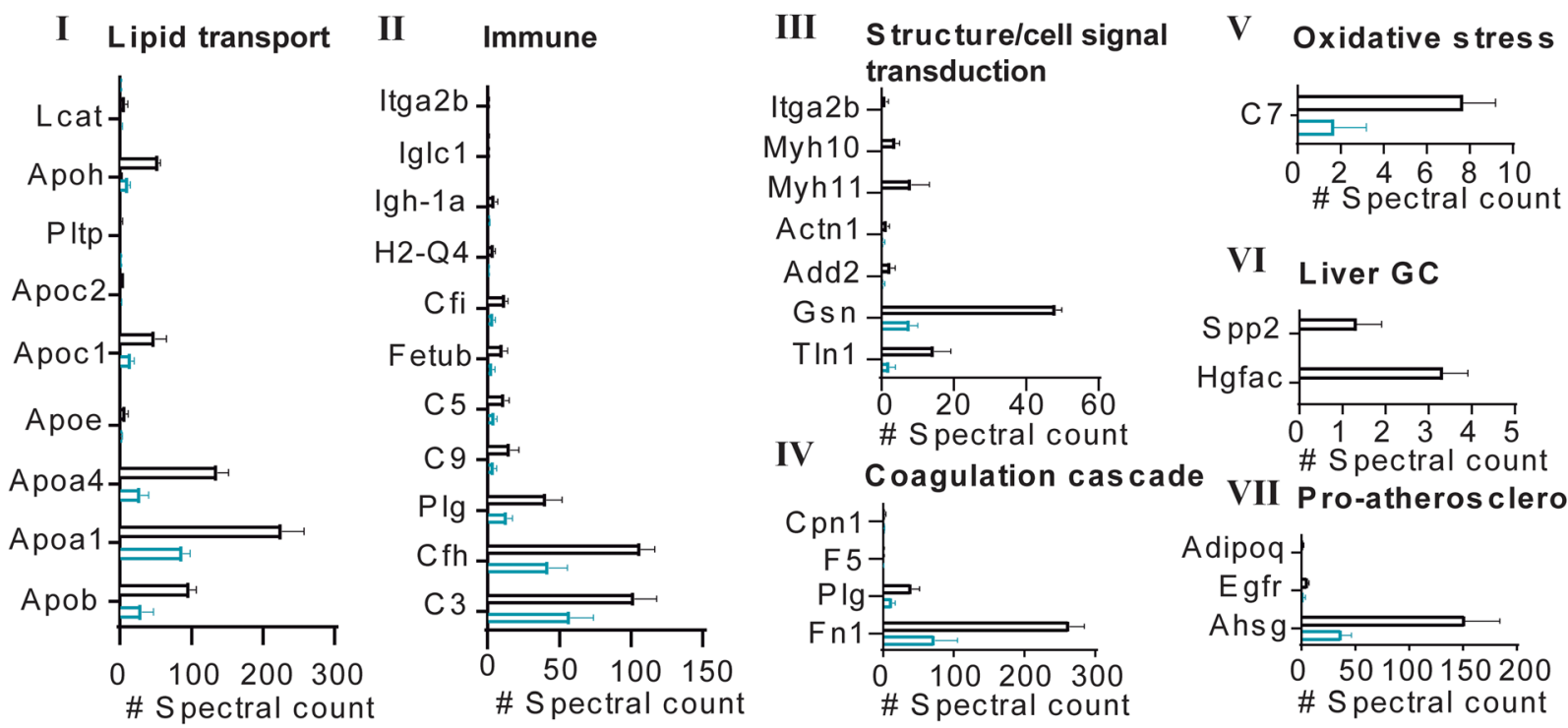

\section{Liver GC}

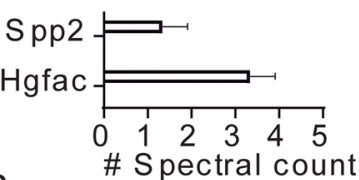

VII Pro-atherosclerotic

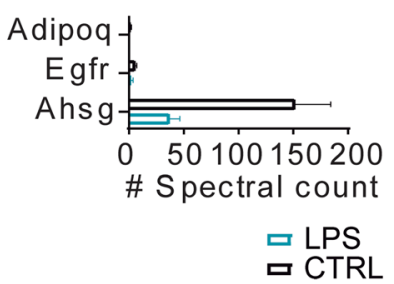

Fig. 3 (See legend on next page.) 
(See figure on previous page.)

Fig. 3 Functional analysis of molecular dynamics in severe inflammatory response for proteins identified from glycocalyx (GC). a Functional categorization of newly synthesized proteins from GC after a severe inflammatory response. $\mathbf{b}$ Relative quantitation of proteins included in the functional categorization. Quantitation of proteins is expressed as spectral counts considering all identified newly synthesized peptides (SILAMlabeled peptides) for every protein. Only proteins with statistical significance assessed by Student's $t$ test are represented $(p<0.05)$

(Ces1c) and transaldose (Taldo1) synthesis (Fig. 4b-II). It is also worth mentioning the downregulation of novel protein synthesis specifically affecting the kidney-related protein cystatin-C (Cst3) in LPS-challenged EC (Fig. 4bVIII).

To a lesser extent, multiple molecular mechanisms altered in EC during Gram-negative sepsis were also similarly affected in SMC, as shown in Fig. 4a. The cellular proteins affected by the impairment of novel protein synthesis include lipid transport-related proteins and proteins implicated in inflammatory processes (Fig. 4a). Signs of specific SMC dysfunction were also evident during Gram-negative sepsis via the downregulation of the synthesis of the proteins vitamin D-binding protein (Gc) and coagulation factor XII (F12) (Fig. 4c-I to IV).

Moreover, we observed the upregulated synthesis of hemopexin, haptoglobin, and serum amyloid proteins in the proteomes of EC in whole organism vascular beds (Fig. 4a, b-I) and of the pro-inflammatory proteins galectin-1 and S100-S9 in SMC together with several histones and serine peptidase inhibitors (Fig. 4a, c-I).

\section{Molecular dynamics of EC phosphoproteomes during Gram-negative sepsis}

The modulation of newly synthesized proteins specifically affected by protein posttranslational modifications (PTMs) during Gram-negative sepsis was also investigated in EC and SMC in whole organism vascular beds. This part of the study revealed that significant modulation of PTMs in newly synthesized proteins was only identified in EC and in proteomes affected by PTM phosphorylation (Fig. 5). Thus, the significant upregulation of the phosphorylation of protein sites was observed in LPS-challenged animals compared to that in sham controls, as shown in Fig. 5. Furthermore, our data indicated that essential EC proteins such as VCAM1 and creatine kinase $\mathrm{M}$-type contain disease-specific phosphorylation sites in Gram-negative sepsis, as detailed in Table 1.

\section{Discussion}

In this work, the combination of SILAM mice [25] with DISDIVO [27] allowed us for the first time to evaluate changes in the molecular dynamics of whole organism vascular beds during severe inflammation linked to Gram-negative sepsis. Our data initially showed the rapid and characteristic disruption and shedding of the pericellular apical coating of the vasculature, known as the GC. This expected finding was in line with that of previous reports [30-33]; however, in this particular case, we additionally found a global decrease in the incorporation of newly synthesized proteins into the GC, which indicates the inhibition of molecular maintenance affecting this vascular coating. Furthermore, negative modulation of phospholipid transfer protein (Pltp) coupled with downregulation of a subset of lipoproteins uncovered specific target molecules contributing to the imbalance in the transfer of essential lipid molecules to this apical vascular layer. Negative regulation of multiple pro-atherosclerotic proteins, complement factors, and plasmatic enzymes was also identified as affecting the GC in our study, which is consistent with previous reports aimed at identifying the molecular basis of GC disruption [34]. The novel data obtained here regarding the molecular dynamics and composition of the GC in Gram-negative sepsis directly advances our knowledge of the role(s) of specific proteins in vascular permeability [35]. The clinical significance of conducting further research aimed at finding novel biological markers that could detect the shedding of the GC was recently pointed out [34]. The GC has the capacity to act as a molecular target for leukocytes and inflammatory mediators, and due to its systemic nature, this mantle is one of the most fragile vascular settings that is highly targeted by endotoxemia, as observed here and in previous reports [27, 31, 36]. The novel-specific proteins linked to shedding and impaired molecular maintenance of the $\mathrm{GC}$, as identified here, require further research to be established as diagnostic/prognostic markers of vascular permeability and endothelial dysfunction in Gramnegative sepsis and in other diseases that involve severe inflammation of the vasculature.

Significant impairment of protein synthesis in the EC and SMC proteomes was also found in this study. Impairment of the molecular dynamics of hindlimb muscle cells was previously reported due to Gram-negative sepsis [37] and has been very recently identified in platelet cells [23]. However, to the best of our knowledge, this has not been previously investigated in whole organism EC and SMC proteomes. Of note, Middleton et al. found highly similar sepsis-induced impairment of protein synthesis in platelet cells between murine and human samples [23]. Similarly, Vary et al. found that sepsis-induced impairment of protein synthesis in platelet cells was associated with the effect of peptide chain initiation and an increased number of free ribosomal subunits in 


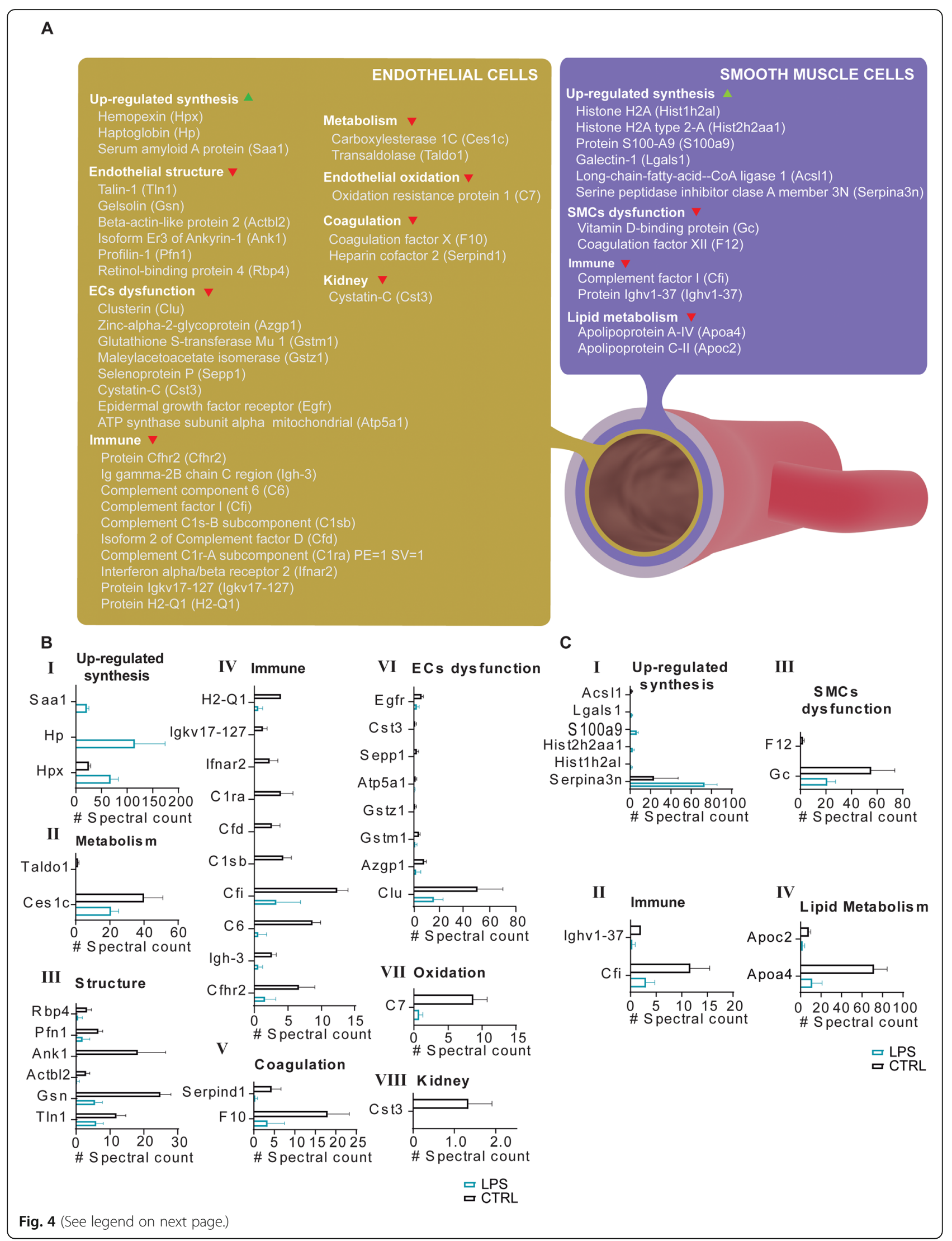


(See figure on previous page.)

Fig. 4 Functional analysis of molecular dynamics in severe inflammatory response for proteins identified from endothelial cells (EC) and smooth muscle cells (SMC). a Functional categorization of newly synthesized proteins identified by DISDIVO after a severe inflammatory response. The relative quantitation of proteins included in the functional categorization for EC and SMC are displayed in sections $\mathbf{b}$ and $\mathbf{c}$, respectively. Quantitation of proteins is expressed as spectral counts considering all identified newly synthesized peptides (SILAM-labeled peptides) for every protein. Only proteins with statistical significance assessed by Student's $t$ test are represented $(p<0.05)$

muscle tissue [37]. Our systems biology approach, as expected, revealed the different mechanisms affecting the molecular dynamics in EC in Gram-negative sepsis. We observed that global protein turnover was significantly upregulated together with the drastic downregulation of protein synthesis affecting EC. These findings in whole organism vascular beds were in line with the catabolic phenotype observed in skeletal muscles from sepsis patients and other critically ill subjects [38]. It has been reported that muscle cells activate the inhibition of protein synthesis together with an increase in protein turnover, which encompasses a progressive and rapid decrease in muscle mass resulting in severe weakness [39]. Nonetheless, although that muscle catabolic phenotype has been described as a consequence of abnormal insulin metabolism and cytokine mediation [40], none of these metabolic processes were yet defined as modulated in the vascular beds of Gram-negative sepsis; with the exception of selenoprotein $\mathrm{P}$, a protein closely related to insulin metabolism [41], which was significantly downregulated in EC, a fact that has been previously associated with the severity of sepsis and other critical illnesses [42, 43].

Careful dissection of the identified catabolic phenotype affecting EC uncovered the involvement of key endothelial-specific proteins such as VCAM1. Activated VCAM1 is directly involved in the transendothelial

\section{Phos phorylation sites}
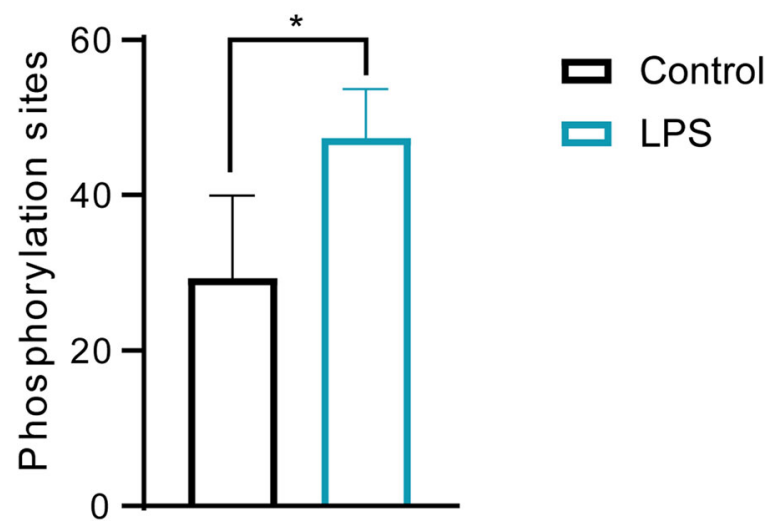

Fig. 5 Number of total phosphorylation sites detected in Gramnegative induced sepsis in EC. The asterisk refers to significant differences observed between groups, assessed by Student's $t$ test $(p<0.05)$ migration of leukocytes [44], and it has been shown that its ubiquitination alters this pro-inflammatory mechanism. Here, we found ongoing direct degradation of VCAM1 at the same time that the protein is potentially activated via disease-specific phosphorylation of the Tyr113 and Ser114 residues, which are located in the region contiguous to the I-7 domain, as shown in Fig. 6. Li et al. [45] recently demonstrated that the degradation of VCAM1 in the pulmonary endothelium is directly linked to improved survival in Gram-negative sepsis. Here, we demonstrate for the first time that this is a systemic process that takes place in whole organism capillary beds in Gram-negative sepsis. This molecular mechanism, as shown in Fig. 6, serves as one of the key pathological mechanisms that sustain/cause severe inflammation in sepsis and potentially in other pathological inflammatory processes affecting the vasculature, a fact that requires follow-up research with potential highly significant clinical implications. In addition to VCAM1, other proteins containing disease-specific phosphorylated sites were also identified in this study. These proteins included spectrin beta chain erythrocytic protein (Sptb) and creatine kinase M-type, among others. Phosphorylation affecting the latter protein, creatine kinase M-type, has been linked to dynamic activation of the protein in the endothelium [46].

Detailed analysis of the molecular dynamics that take place during Gram-negative sepsis also confirmed the upregulation of the synthesis of serum amyloid A protein (Saa1) as one of the main inflammatory proteins activated in EC. This severe phase protein is generally elevated in blood during inflammation [47]. Multiple prothrombotic proteins were also upregulated in vascular beds together with the downregulation of various coagulation cascade-related proteins, including the coagulation factors F10 and F12. Activation of inflammatory and microthrombotic mediators, as encountered in EC in this study, is closely linked to sepsis endotheliopathy and seems to lead to the development of a series of fatal conditions, including thrombocytopenia, microangiopathic hemolytic anemia, and multiorgan dysfunction syndrome [39]. In addition, our protein dynamics investigation also indicated the activation of multiple protective innate mechanisms, particularly in EC. These innate protective mechanisms included the upregulation of the antithrombotic heme-binding plasma glycoprotein hemopexin $(\mathrm{Hpx})$ [48] and the upregulation of the anti- 
Table 1 Phosphorylated proteins identified exclusively in Gram-negative sepsis in EC proteome. *Information about domains and structure for every protein was obtained from Uniprot. Numbers in brackets indicate the localization of the protein regions referred based on the amino acids' position in the protein sequence

\begin{tabular}{llll}
\hline Gene symbol & Protein name & Modified residue & Post-translationally modified region* \\
\hline Sptb & Spectrin beta chain erythrocytic & S1061, S1078, S2323 & - \\
$\mathrm{Cp}$ & Ceruloplasmin & T83 & Chain (20-1061), F5/8 type A 1 Domain (20-356), Plastocyanin-like 1 (20-199) \\
$\mathrm{Hp}$ & Haptoglobin & $\mathrm{S} 210, \mathrm{~S} 239$ & Polypeptide chain (19-347), Peptidase S1 domain (103-345) \\
$\mathrm{Ckm}$ & Creatine kinase M-type & $\mathrm{T} 208, \mathrm{~S} 224$ & Phosphagen kinase C-terminal domain (125-367) \\
Nsfl1C & Isoform 3 of NSFL1 cofactor p47 & $\mathrm{T} 108, \mathrm{~S} 116$ & Before and after the nuclear localization signal motif (109-115) \\
Serpina1d & Alpha-1-antitrypsin 1-4 & $\mathrm{S} 300$ & Alpha-1-antitrypsin 1-4 Chain (25-413) \\
Vcam1 & Vascular cell adhesion protein 1 & $\mathrm{Y} 113, \mathrm{~S} 114$ & Extracellular domain (25-698) -next to I-set domain (C-terminal) \\
\hline
\end{tabular}

inflammatory acute phase protein haptoglobin (Hp) [49] coupled to the downregulation of the anti-thrombotic and immune-related protein heparin cofactor 2 (Serpind1). The protective capacity of Serpind1 was associated with the presence of proteolytic fragments of the protein with antimicrobial and anti-coagulant capacities [50]; such fragments were also encountered in this study and linked to the downregulation of the protein, a fact that further confirms the protective and compensatory nature of the finding in whole organism capillary beds during Gram-negative sepsis. Furthermore, we consider that confirmation of the protective nature of the proteolytic Serpind1 fragments identified here paves the way for future systemic therapeutic interventions for sepsis using specific Serpind1 fragments, which requires specific investigation based on the findings reported here.

Impairment in the molecular dynamics of whole organism vascular beds also affected SMC, which is in line with the described effects on EC, although the effect on SMC was observed to a lesser extent. Downregulation of the synthesis of key proteins, such as vitamin D binding protein and coagulation factor XII, was encountered in SMC proteomes. This fact, however, further confirms that Gram-negative sepsis has major effects on whole organism capillary beds, which explains the exacerbated effect on the molecular dynamics of EC compared with that of SMC.

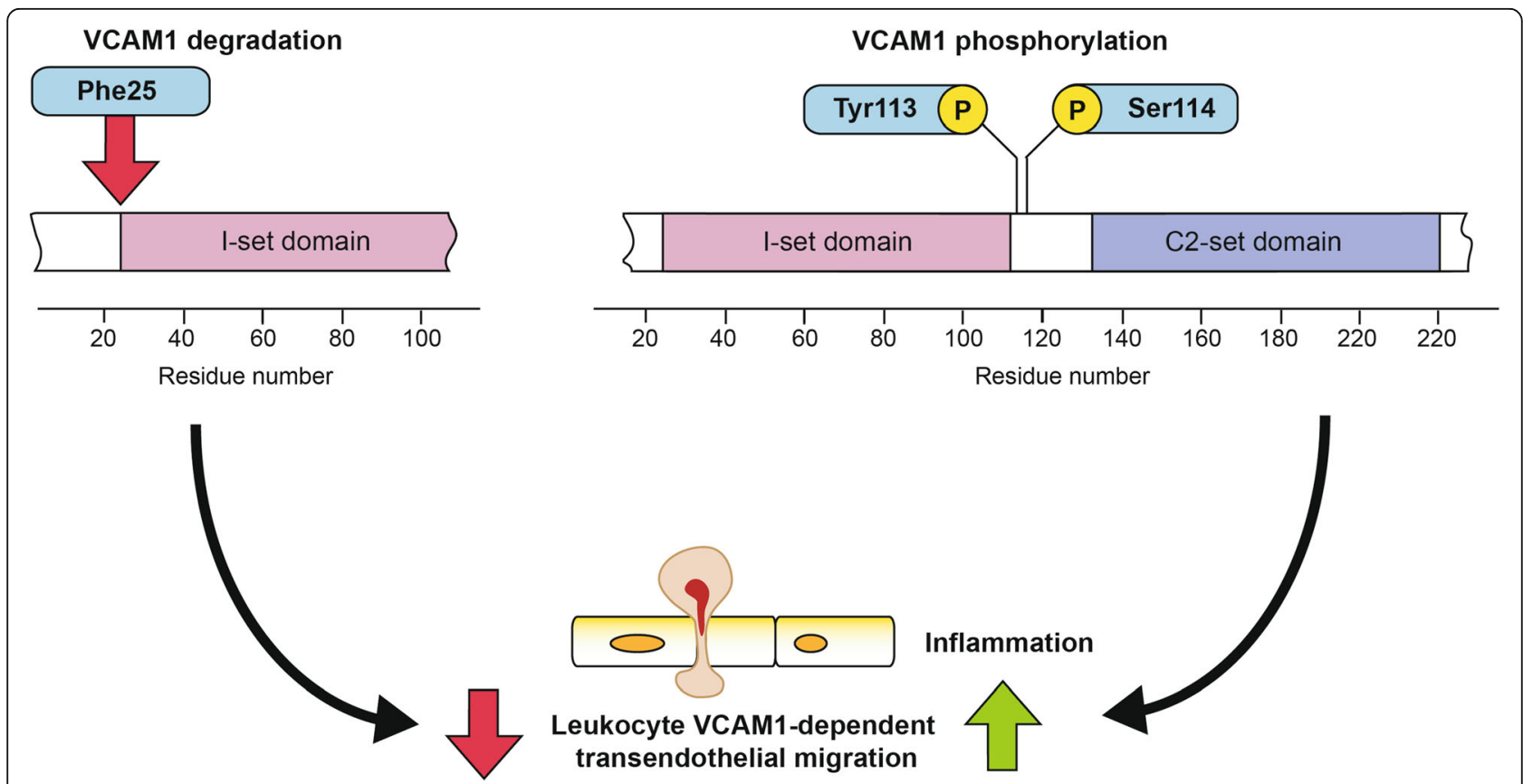

Fig. 6 Illustrative diagram showing the identified inflammatory molecular mechanisms of VCAM1 in EC during Gram-negative induced sepsis. VCAM1 in EC contains disease-specific phosphorylations at Tyr113 and Ser114 in pro-inflammatory processes of Gram-negative-induced sepsis. Additionally, the protein is actively degraded at Phe25, which might indicate resilience of EC during systemic pro-inflammatory processes affecting the vasculature 


\section{Conclusions}

Gram-negative sepsis has been widely used to model sepsis, and it is accepted that LPS exposure is an important part of this complex illness. Thus, our generated data expand on the previously limited knowledge about how protein synthesis and degradation become altered in the vasculature due to a systemic inflammatory response in sepsis. Globally, our novel generated data indicate that proteome molecular dynamics become altered in EC with a major impact on whole organism capillary beds. Similarly, EC fail to maintain the proper molecular integrity of the $\mathrm{GC}$ by not providing that apical layer with the required newly synthesized structural proteins. Furthermore, abnormal protein turnover during Gramnegative sepsis affects essential EC proteins, such as VCAM1, and is coupled to the global downregulation of protein synthesis and the generation of disease-specific phosphorylation sites. Finally, SMC alter their molecular dynamics in line with EC, although to a lesser extent.

The findings reported here, thus, uncover for the first time specific molecules that become altered in the protein synthesis machinery of the GC and EC, which indicates that the increase in VCAM1 that is typically associated with endothelial dysfunction in several diseases, including sepsis, may be due to altered degradation of the protein and the accumulation in the endothelium of dysfunctional VCAM1. Similarly, novel Gram-negative sepsis-specific phosphorylation sites have been uncovered for the first time. These findings can serve as a foundation for future therapeutic strategies aimed at maintaining the structural and functional integrity of the vasculature in sepsis, as they can provide novel insights into the previously unknown molecular mechanisms that become altered in the vasculature due to the systemic inflammatory response, which is a pathological mechanism common to several human diseases.

\section{Methods \\ Reagents}

All reagents were purchased from Sigma-Aldrich (St. Louis, MO) unless otherwise specified. Sequencinggrade modified trypsin was purchased from Promega (Madison, WI).

\section{Animals}

Ten-week-old male C57BL/6NT mice were housed in cages on a 12-h dark/light cycle at stable temperature $\left(21^{\circ} \mathrm{C}\right)$ with water provided ad libitum and fed with standard commercial chow for a minimum of 2 weeks (adaptation period) before starting the experimental part. All experimental procedures were approved by the Nanyang Technological University Institutional Animal Care and Use Committee (IACUC) and were performed humanely and in strict accordance with the International
Guiding Principles for Animal Research. The 3Rs principle in animal experimentation [51] was in all cases observed.

\section{Sepsis-induced inflammation model under stable isotope- labeled diet}

Mice were maintained in fasting conditions for $16 \mathrm{~h}$ before being exposed to a stable isotope-labeled diet (Lys(6)-SILAM-Mouse diet, pellet $\varnothing 10 \mathrm{~mm}$, Silantes $\mathrm{GmbH}$, München, Germany; $n=6$ ). As the effect potentially caused by an acute treatment with LPS on the protein dynamics of the endothelium was expected to be strong, as it was later identified, we kept the number of animals used in the experiments to the minimum that allowed to identify outliers and to obtain statistical significance, as previously recommended [52]. Mice were kept in SILAM diet for $24 \mathrm{~h}$ before treatment and were then divided into 2 groups (control and LPS treated). LPS treated mice were injected with a total of $20 \mathrm{mg} / \mathrm{kg}$ of lipopolysaccharide [27, 53] derived from Escherichia coli O55:B5 freshly prepared in sterile PBS (vehicle solution). LPS was administered in two equal doses of 10 $\mathrm{mg} / \mathrm{kg}$ in a $24 \mathrm{~h}$ interval to ensure proper intake of SILA $M$ stable isotope-labeled chow. LPS mice were maintained in SILAM diet during all experimental procedures. Similarly, control mice were injected with vehicle solution in a 24-h interval and maintained in SILAM diet during the whole experimental procedures.

\section{DISDIVO obtention of whole organism vascular beds}

Systemic isolation of vascular beds was carried out by differential systemic decellularization in vivo (DISDIVO) as previously described [27]. Briefly, mice were anesthetized by intraperitoneal injection with ketamine-xylazine (90:10 $\mathrm{mg} / \mathrm{kg}$ ) and deep anesthesia was maintained over the whole experimental procedure by inhalation of isoflurane (IsoFlo; Veterinaria Esteve, Bologna, Italy). For the DISDIVO procedure, an exsanguination by transcardial whole-body perfusion with open right auricle was performed while $1 \times$ PBS was simultaneously introduced at a flow rate of $1.5 \mathrm{~mL} / \mathrm{min}$ through the left ventricle. PBS perfusion was maintained for $1.5 \mathrm{~min}$ after complete removal of blood when the collection of PBS fraction from the open right auricle was initiated and maintained over 3 to $4 \mathrm{~min}$ to collect the GC-containing outflow. EC decellularization was subsequently performed perfusing with $0.5 \%$ sodium deoxycholate (SDC) prepared in $100 \mathrm{mmol} / \mathrm{L}$ ammonium acetate buffer through the entire circulatory system. EC decellularization was maintained over 3-4 min collecting the EC lysed tissuecontaining outflow from the open right auricle, and subsequently, concentration of SDC was increased to $10 \%$ to decellularize SMC vascular beds. Perfusion with $10 \%$ SDC prepared in $100 \mathrm{mM}$ ammonium acetate buffer was 
maintained over 3-4 min. All collected outflows were stored at $-80^{\circ} \mathrm{C}$ until analysis.

In-solution tryptic digestion of vascular beds proteomes Vascular beds proteomes were digested by in-solution digestion as previously described [27, 54]. Briefly, DISDIVO outflows were adjusted to $1 \%$ SDC using a $10 \%$ SDC stock solution prepared in $100 \mathrm{mM}$ ammonium acetate for GC and EC fractions or by dilution with $100 \mathrm{mM}$ ammonium acetate for SMC fractions. Vascular beds proteins were subsequently reduced using $10 \mathrm{mmol} / \mathrm{L}$ dithiothreitol (DTT) for $30 \mathrm{~min}$ at $60{ }^{\circ} \mathrm{C}$ and alkylated using $20 \mathrm{mmol} / \mathrm{L}$ iodoacetamide for $45 \mathrm{~min}$ at room temperature protected from the light. Samples were then 2-fold diluted with $10 \mathrm{mmol} /$ L DTT prepared in $100 \mathrm{mmol} / \mathrm{L}$ ammonium acetate and incubated for $30 \mathrm{~min}$ at $37^{\circ} \mathrm{C}$. Tryptic digestion was performed at $30^{\circ} \mathrm{C}$ overnight using sequencinggrade-modified trypsin at 1:50 (w/w) enzyme-toprotein ratio. Enzymatic digestion was quenched by addition of a final concentration of $0.5 \%$ formic acid (FA) and SDC salts were precipitated by acidification. Peptide recovery from precipitated SDC was performed as follows: SDC was pelleted by centrifugation at $12,000 \mathrm{~g}$ for $10 \mathrm{~min}$ at $4{ }^{\circ} \mathrm{C}$. The supernatant containing peptides was then separated and pelleted SDC was redissolved in $0.5 \%$ ammonium hydroxide before reprecipitation with $0.5 \%$ FA. Peptide recovery was performed per duplicate and supernatant combined. Peptides were desalted using a C18 Sep-pack cartridge (Waters, Milford, MA). Eluates were finally dried in a vacuum concentrator (Eppendorf, Hamburg, Germany).

\section{High-pressure liquid chromatography fractionation of vascular beds proteomes}

Vascular beds desalted peptides were fractionated by high-pressure liquid chromatography as previously described [55]. Briefly, dried samples were reconstituted in $200 \mu \mathrm{L}$ of $10 \mathrm{mmol} / \mathrm{L}$ ammonium hydroxide in water (mobile phase A) and separated using a XBridge BEH130 C18, $3.5 \mu \mathrm{m}, 4.6 \times 250 \mathrm{~mm}$ column (Waters, Elstree, UK) on a Shimadzu Prominence UFLC system (Dionex, Sunnyvale, CA) monitoring UV of peptide intensities at $280 \mathrm{~nm}$. Peptide separation was performed over a 72 -min gradient at $1 \mathrm{~mL} / \mathrm{min}$ as follows: $0 \%$ mobile phase $\mathrm{B}(10 \mathrm{mmol} / \mathrm{L}$ ammonium hydroxide in acetonitrile) for $5 \mathrm{~min}, 0 \%$ to $20 \%$ for $30 \mathrm{~min}, 20 \%$ to $33 \%$ for $15 \mathrm{~min}, 33 \%$ to $60 \%$ for $10 \mathrm{~min}$, and $60 \%$ to $100 \%$ for 5 min, followed by $7 \mathrm{~min}$ at $0 \%$ mobile phase B. Fractions were collected every minute and combined by concatenation. Combined fractions were completely dried in the vacuum concentrator.

\section{Liquid chromatography tandem-mass spectrometry} analysis of vascular beds proteomes

Dried fractionated peptides were reconstituted in 3\% acetonitrile $(\mathrm{ACN}), 0.1 \%$ FA (mobile phase A), and analyzed by liquid chromatography tandem-mass spectrometry (LC-MS/MS) using a Dionex UltiMate 3000 UHPLC system coupled with an Orbitrap Elite mass spectrometer (Thermo Fisher, Inc., Bremen, Germany) [56, 57]. The sample was sprayed using a Thermo Fisher Easy-Spray source working at $1.5 \mathrm{kV}$ and separated using a reverse-phase Acclaim PepMap RSL column $(75 \mu \mathrm{m}$ ID $\times 15 \mathrm{~cm}, 2-\mu \mathrm{m}$ particle size; Thermo Scientific, Inc.) maintained at $35^{\circ} \mathrm{C}$ and working at $300 \mathrm{~nL} / \mathrm{min}$. Peptides were separated over a 60-min gradient as follows: $3 \%$ mobile phase B (90\% acetonitrile, $0.1 \% \mathrm{FA}$ ) for $1 \mathrm{~min}, 3 \%$ to $35 \%$ for $47 \mathrm{~min}, 35 \%$ to $50 \%$ for $4 \mathrm{~min}, 80 \%$ for $6 \mathrm{~s}, 80 \%$ (isocratic) for $78 \mathrm{~s}, 80 \%$ to $3 \%$ for $6 \mathrm{~s}$, and then maintained at $3 \%$ (isocratic) for $6.5 \mathrm{~min}$. Data adquisition using Xcalibur 2.2 SP1.48 software (Thermo Fisher Inc., Bremen, Germany) was performed in positive mode alternating between full Fourier transform mass spectrometry (FT-MS; 350-2000 m/z, resolution 60, 000, $1 \mu$ scan per spectrum) and FT-MS/MS (150$2000 \mathrm{~m} / \mathrm{z}$, resolution 30,000, $1 \mu$ scan per spectrum). The 10-most intense ions with charge $>+2$ were isolated within a 2-Da window and fragmented by highenergy collisional dissociation mode using 32\% normalized collision energy with a threshold of 500 counts. Automatic gain control was set to $1 \times 10^{6}$ for FT-MS and FT-MS/MS.

\section{Bioinformatics and data analysis}

Database search of raw proteomics data obtained from the LC-MS/MS analysis was analyzed using PEAKS Studio version 7.526 (Bioinformatics Solutions, Waterloo, Canada) as previously described [58, 59] with minor modifications. The database search was performed using an ion tolerance of $10 \mathrm{ppm}$ and a fragment ion tolerance of $0.05 \mathrm{Da}$. The false-discovery rate used was 1\% [60]. Carbamidomethylation at Cys was set as fixed modification and SILAC K6 $(+6.0201 \mathrm{Da})$ at Lys was set as variable modification. The UniProt mouse database (58,761 entries; downloaded on February 18, 2016) was used for searching. Only proteins consistently identified in at least 2 animals were considered. Identification of protein posttranslational modifications (PTMs) was carried out using PEAKS PTM algorithm and only PTM manually validated and with an Ascore of 1000 were considered in this study. Obtained raw data were analyzed in Microsoft Excel with the help of in-house created macros. GraphPad Prism 8 (GraphPad Software, Palo Alto, CA) was used for statistical analyses of results 
and creation of data plots. Statistical significance was established by ANOVA followed by Bonferroni post hoc multiple comparisons at $P<0.05$, unless otherwise specified. Data are reported as mean $\pm \mathrm{SD}$, unless stated otherwise. Illustrations were created using the open-source software Blender version 2.8 [61] and Adobe Illustrator CS5.

\section{Supplementary Information}

Supplementary information accompanies this paper at https://doi.org/10 1186/s12915-020-00914-0.

Additional file 1. List of peptides identified in DISDIVO-isolated vascular beds (GC, ECs and SMCs) from LPS-treated mice. Replicate for every vascular bed analyzed are included in different Tabs (1 to 3)

Additional file 2. List of peptides identified in DISDIVO-isolated vascular beds (GC, ECs and SMCs) from Control mice. Replicate for every vascular bed analyzed are included in different Tabs (1 to 3).

\section{Abbreviations}

ACN: Acetonitrile; CVS: Cardiovascular system; DISDIVO: Differential systemic decellularization in vivo; DTT: Dithiothreitol; EC: Endothelial cells; FA: Formic acid; GC: Glycocalyx; LC-MS/MS: Liquid chromatography tandem-mass spectrometry; LPS: Lipopolysaccharide; NSP: Newly synthesized proteins; NNSP: Non-newly synthesized proteins; PTM: Post-translational modifications; SDC: Sodium deoxycholate; SILAM: Stable isotope labeling of mammals; SMC: Smooth muscle cells

\section{Acknowledgements}

We thank Paul Gamboa and Esther Veronica Wong and the rest of the staff from the Animal Research Facility of the Lee Kong Chian School of Medicine, Nanyang Technological University, Singapore, for their kind help and support with the animal experiments performed in this work. The authors thank Cristina Lorca from IMDEA Food \& Health Sciences Research Institute for her help on the proofreading of the manuscript. We also thank Francisco Arcones from the IT team of IMDEA Food \& Health Sciences Research Institute for his kind help with the workstation and platform set up.

\section{Authors' contributions}

X.G-P and A.S. contributed to the experimental part, analysis of obtained data, and drafting of the manuscript. A.S. and S.K.S. contributed with important and critical intellectual content to the whole study and manuscript draft. All authors approved the final version of the manuscript for submission.

\section{Funding}

Support for this work was provided by the National Medical Research Council of Singapore (NMRC-OF-IRG-0003-2016), Ministry of Education of Singapore (MOE2016-T2-2-018), and the Research and Education Council of the Comunidad de Madrid, Spain (2018-T1/BIO-10633). Dr. Aida Serra acknowledges a grant from the Talento Program 2018 of the Comunidad de Madrid, and Dr. Xavier Gallart-Palau acknowledges a grant from the Sara Borrell Program (CD19/00243) of the Carlos III Institute of Health, Ministry of Economy and Competitiveness (Spain), awarded on the 2019 call under the Health Strategy Action 2017-2020 (This grant is co-funded with European Union ERDF Funds (European Regional Development Fund)).

\section{Availability of data and materials}

All data generated in this study have been made publicly available as indicated below:

Authors: Xavier Gallart-Palau, Aida Serra, Siu Kwan Sze

Publisher: ProteomeXchange via repository PRIDE

Title: Characterization of proteome dynamics in individual vascular layers at the early stage of acute sepsis

Accession: PXD018274

Project Webpage: http://www.ebi.ac.uk/pride/archive/projects/PXD018274 FTP Download: ftp://ftp.pride.ebi.ac.uk/pride/data/archive/2020/10/PXD0182 74

\section{Ethics approval and consent to participate}

All experimental procedures were approved by the Nanyang Technological University Institutional Animal Care and Use Committee (IACUC) and were performed humanely and in strict accordance with the International Guiding Principles for Animal Research.

\section{Consent for publication}

Not applicable.

\section{Competing interests}

The authors declare that they have no competing interests with regards of the data and conclusions reported.

\section{Author details}

${ }^{1}$ School of Biological Sciences, Nanyang Technological University, 60 Nanyang Drive, Singapore 637551, Singapore. ${ }^{2}$ University Hospital Institut Pere Mata, Reus, Tarragona, Spain. ${ }^{3}$ Institut Investigació Sanitària Pere Virgili (IISPV), Reus, Tarragona, Spain. ${ }^{4}$ Centro de investigación Biomédica en Salud Mental CIBERSAM, Instituto de Salud Carlos III, Madrid, Spain. ${ }^{5}$ IMDEA Food \& Health Sciences Research Institute, +Pec Proteomics, Campus of International Excellence UAM+CSIC, Old Cantoblanco Hospital, 8 Crta. Canto Blanco, 28049 Madrid, Spain. ${ }^{6}$ Proteored - Instituto de Salud Carlos III (ISCIII), Madrid, Spain.

Received: 5 October 2020 Accepted: 3 November 2020

Published online: 24 November 2020

\section{References}

1. Minami T, Muramatsu M, Kume T. Organ/tissue-specific vascular endothelial cell heterogeneity in health and disease. Biol Pharm Bull. 2019:42(10):1609-19.

2. Rushmer RF. Structure and function of the cardiovascular system. In: Schneiderman N, Weiss SM, Kaufmann PG, editors. Handbook of Research Methods in Cardiovascular Behavioral Medicine. Boston: Springer US; 1989 p. 5-22.

3. Fisher SA. Vascular smooth muscle phenotypic diversity and function. Physiol Genomics. 2010:42A(3):169-87.

4. Alarcon-Martinez L, Yilmaz-Ozcan S, Yemisci M, Schallek J, Kılıç K, Can A, Di Polo A, Dalkara T. Capillary pericytes express a-smooth muscle actin, which requires prevention of filamentous-actin depolymerization for detection. eLife. 2018;7:e34861

5. Wimmer RA, Leopoldi A, Aichinger M, Wick N, Hantusch B, Novatchkova M, Taubenschmid J, Hammerle M, Esk C, Bagley JA, et al. Human blood vessel organoids as a model of diabetic vasculopathy. Nature. 2019; 565(7740):505-10

6. Colbert JF, Schmidt EP. Endothelial and microcirculatory function and dysfunction in sepsis. Clin Chest Med. 2016;37(2):263-75.

7. Gallart-Palau X, Serra A, Hase Y, Tan CF, Chen CP, Kalaria RN, Sze SK. Brainderived and circulating vesicle profiles indicate neurovascular unit dysfunction in early Alzheimer's disease. Brain Pathol. 2019;29(5):593-605.

8. Poisson J, Tanguy M, Davy H, Camara F, El Mdawar MB, Kheloufi M, Dagher T, Devue C, Lasselin J, Plessier A, et al. Erythrocyte-derived microvesicles induce arterial spasms in JAK2V617F myeloproliferative neoplasm. J Clin Invest. 2020;130(5):2630-43.

9. Park L, Uekawa K, Garcia-Bonilla L, Koizumi K, Murphy M, Pistik R, Younkin L, Younkin S, Zhou P, Carlson G, et al. Brain perivascular macrophages initiate the neurovascular dysfunction of Alzheimer Abeta peptides. Circ Res. 2017; 121(3):258-69.

10. Gallart-Palau X, Guo X, Serra A, Sze SK. Alzheimer's disease progression characterized by alterations in the molecular profiles and biogenesis of brain extracellular vesicles. Alzheimers Res Ther. 2020;12(1):54.

11. Derada Troletti C, Fontijn RD, Gowing E, Charabati M, van Het Hof B, Didouh I, van der Pol SMA, Geerts D, Prat A, van Horssen J, et al. Inflammation-induced endothelial to mesenchymal transition promotes brain endothelial cell dysfunction and occurs during multiple sclerosis pathophysiology. Cell Death Dis. 2019;10(2):45.

12. Rajendran $P$, Rengarajan T, Thangavel J, Nishigaki Y, Sakthisekaran D, Sethi G, Nishigaki I. The vascular endothelium and human diseases. Int J Biol Sci. 2013;9(10):1057-69.

13. Xiao F, Wang D, Kong L, Li M, Feng Z, Shuai B, Wang L, Wei Yg, Li H, Wu S et al: Intermedin protects against sepsis by concurrently re-establishing the 
endothelial barrier and alleviating inflammatory responses. Nat Commun 2018, 9(1):2644.

14. Kempker JA, Martin GS. A global accounting of sepsis. Lancet. 2020; 395(10219):168-70

15. Rudd KE, Johnson SC, Agesa KM, Shackelford KA, Tsoi D, Kievlan DR, Colombara DV, Ikuta KS, Kissoon N, Finfer S, et al. Global, regional, and national sepsis incidence and mortality, 1990-2017: analysis for the Global Burden of Disease Study. Lancet. 2020;395(10219):200-11.

16. Opal SM, Scannon PJ, Vincent J-L, White M, Carroll SF, Palardy JE, Parejo NA, Pribble JP, Lemke JH. Relationship between plasma levels of lipopolysaccharide (LPS) and LPS-binding protein in patients with severe sepsis and septic shock. J Infect Dis. 1999;180(5):1584-9.

17. Chen KF, Chaou CH, Jiang JY, Yu HW, Meng YH, Tang WC, Wu CC. Diagnostic accuracy of lipopolysaccharide-binding protein as biomarker for sepsis in adult patients: a systematic review and meta-analysis. PLoS One. 2016;11(4):e0153188.

18. Zanoni I, Ostuni R, Barresi S, Di Gioia M, Broggi A, Costa B, Marzi R, Granucci F. CD14 and NFAT mediate lipopolysaccharide-induced skin edema formation in mice. J Clin Invest. 2012;122(5):1747-57.

19. Bosmann M, Ward PA. The inflammatory response in sepsis. Trends Immunol. 2013;34(3):129-36.

20. Libert C, Ayala A, Bauer M, Cavaillon JM, Deutschman C, Frostell C, Knapp S, Kozlov AV, Wang P, Osuchowski MF, et al. Part II: Minimum Quality Threshold in Preclinical Sepsis Studies (MQTiPSS) for types of infections and organ dysfunction endpoints. Shock. 2019;51(1):23-32.

21. Le Quesne JP, Spriggs KA, Bushell M, Willis AE. Dysregulation of protein synthesis and disease. J Pathol. 2010;220(2):140-51.

22. Scheper GC, van der Knaap MS, Proud CG. Translation matters: protein synthesis defects in inherited disease. Nat Rev Genet. 2007:8(9):711-23.

23. Middleton EA, Rowley JW, Campbell RA, Grissom CK, Brown SM, Beesley SJ, Schwertz H, Kosaka Y, Manne BK, Krauel K, et al. Sepsis alters the transcriptional and translational landscape of human and murine platelets. Blood. 2019;134(12):911-23.

24. Ten Have GAM, Engelen M, Wolfe RR, Deutz NEP. Inhibition of jejunal protein synthesis and breakdown in Pseudomonas aeruginosa-induced sepsis pig model. Am J Physiol-Gastrol L. 2019;316(6):G755-g762.

25. McClatchy DB, Yates JR 3rd. Stable isotope labeling in mammals (SILAM). Methods Mol Biol. 2014;1156:133-46.

26. Rauniyar N, McClatchy DB, Yates JR 3rd. Stable isotope labeling of mammals (SILAM) for in vivo quantitative proteomic analysis. Methods. 2013;61(3): 260-8.

27. Serra A, Gallart-Palau X, Park JE, Lim GGY, Lim KL, Ho HH, Tam JP, Sze SK. Vascular bed molecular profiling by differential systemic decellularization in vivo. Arterioscler Thromb Vasc Biol. 2018;38(10): 2396-409.

28. Wu CC, MacCoss MJ, Howell KE, Matthews DE, Yates JR 3rd. Metabolic labeling of mammalian organisms with stable isotopes for quantitative proteomic analysis. Anal Chem. 2004;76(17):4951-9.

29. Lang F, Aravamudhan S, Nolte H, Turk C, Holper S, Muller S, Gunther S, Blaauw B, Braun T, Kruger M. Dynamic changes in the mouse skeletal muscle proteome during denervation-induced atrophy. Dis Models Mech. 2017;10(7):881-96.

30. Ince C, Mayeux PR, Nguyen T, Gomez H, Kellum JA, Ospina-Tascon GA, Hernandez G, Murray P, De Backer D. The endothelium in sepsis. Shock. 2016;45(3):259-70.

31. Ushiyama A, Kataoka H, lijima T. Glycocalyx and its involvement in clinical pathophysiologies. J Intensive Care. 2016;4(1):59.

32. Vlahu CA, Krediet RT. Can plasma hyaluronan and hyaluronidase be used as markers of the endothelial glycocalyx state in patients with kidney disease? Adv Perit Dial. 2015:31:3-6.

33. Smart L, Macdonald SPJ, Burrows S, Bosio E, Arendts G, Fatovich DM. Endothelial glycocalyx biomarkers increase in patients with infection during Emergency Department treatment. J Crit Care. 2017:42:304-9.

34. Iba T, Levy JH. Derangement of the endothelial glycocalyx in sepsis. J Thromb Haemost. 2019;17(2):283-94.

35. Chelazzi C, Villa G, Mancinelli P, De Gaudio AR, Adembri C. Glycocalyx and sepsis-induced alterations in vascular permeability. Crit Care. 2015;19(1):26

36. Inagawa R, Okada H, Takemura G, Suzuki K, Takada C, Yano H, Ando Y, Usui T, Hotta Y, Miyazaki N, et al. Ultrastructural alteration of pulmonary capillary endothelial glycocalyx during endotoxemia. Chest. 2018;154(2): $317-25$.
37. Vary TC, Kimball SR. Sepsis-induced changes in protein synthesis: differential effects on fast- and slow-twitch muscles. Am J Phys. 1992;262(6 Pt 1):C1513-9.

38. Vary TC. Regulation of skeletal muscle protein turnover during sepsis. Curr Opin Clin Nutr Metab Care. 1998:1 (2):217-24.

39. Cankayali I, Boyacilar O, Demirag K, Uyar M, Moral AR. Neuromuscular dysfunction in experimental sepsis and glutamine. Balkan Med J. 2016;33(3):267-74.

40. Casas RMLZR, Pasquetti CA, Meléndez MG. Protein turnover in sepsis. Rev Endocrinol Nutr. 2003;11(3):136-41.

41. Mao J, Teng W. The relationship between selenoprotein P and glucose metabolism in experimental studies. Nutrients. 2013;5(6):1937-48.

42. Zhao Y, Banerjee $S$, Huang $P$, Wang $X$, Gladson CL, Heston WD, Foster CB. Selenoprotein P neutralizes lipopolysaccharide and participates in hepatic cell endoplasmic reticulum stress response. FEBS Lett. 2016;590(24):4519-30.

43. Hollenbach B, Morgenthaler NG, Struck J, Alonso C, Bergmann A, Kohrle J, Schomburg L. New assay for the measurement of selenoprotein $P$ as a sepsis biomarker from serum. J Trace Elem Med Biol. 2008;22(1):24-32.

44. Weber C. Involvement of tyrosine phosphorylation in endothelial adhesion molecule induction. Immunol Res. 1996;15(1):30-7.

45. Li Y, Huang X, Guo F, Lei T, Li S, Monaghan-Nichols P, Jiang Z, Xin HB, Fu M. TRIM65 E3 ligase targets VCAM-1 degradation to limit LPS-induced lung inflammation. J Mol Cell Biol. 2020;12(3):190-201.

46. Palmer AK, Fraga D, Edmiston PL. Regulation of creatine kinase activity by phosphorylation of serine-199 by AMP-activated kinase. FASEB JI. 2008;22(1_ supplement):1012.1010.

47. Moran G, Carcamo C, Concha M, Folch H. Expression of the protein serum amyloid $\mathrm{A}$ in response to Aspergillus fumigatus in murine models of allergic airway inflammation. Rev Iberoam Micol. 2015;32(1):25-9.

48. Hassaan P, Mehanna R, Dief A. The potential role of hemopexin and heme oxygenase-1 inducer in a model of sepsis. Physiol J. 2015;2015:1-10.

49. Wang Y, Kinzie E, Berger FG, Lim SK, Baumann H. Haptoglobin, an inflammation-inducible plasma protein. Redox Rep. 2001;6(6):379-85.

50. Kalle M, Papareddy P, Kasetty G, Tollefsen DM, Malmsten M, Morgelin M, Schmidtchen A. Proteolytic activation transforms heparin cofactor II into a host defense molecule. J Immunol. 2013;190(12):6303-10.

51. MacArthur Clark J. The 3Rs in research: a contemporary approach to replacement, reduction and refinement. Br J Nutr. 2018;120(s1):S1-s7.

52. Goñi R, Garcia P, Foissac S: The qPCR data statistical analysis. In: Integromics: 2009; Tres cantos. Integromics White Paper: 1-9.

53. Ehrentraut S, Frede S, Stapel H, Mengden T, Grohe C, Fandrey J, Meyer R, Baumgarten G. Antagonism of lipopolysaccharide-induced blood pressure attenuation and vascular contractility. Arterioscler Thromb Vasc Biol. 2007; 27(10):2170-6.

54. Serra A, Zhu H, Gallart-Palau X, Park JE, Ho HH, Tam JP, Sze SK. Plasma proteome coverage is increased by unique peptide recovery from sodium deoxycholate precipitate. Anal Bioanal Chem. 2016;408:1963-73.

55. Gallart-Palau X, Serra A, Lee BST, Guo X, Sze SK. Brain ureido degenerative protein modifications are associated with neuroinflammation and proteinopathy in Alzheimer's disease with cerebrovascular disease. $J$ Nneuroinflammation. 2017;14(1):175.

56. Gallart-Palau X, Serra A, Sze SK. Enrichment of extracellular vesicles from tissues of the central nervous system by PROSPR. Mol Neurodegener. 2016;1 1(1):41.

57. Gallart-Palau X, Serra A, Wong ASW, Sandin S, Lai MKP, Chen CP, Kon OL, Sze SK. Extracellular vesicles are rapidly purified from human plasma by PRotein Organic Solvent PRecipitation (PROSPR). Sci Rep. 2015;5: Article number: 14664.

58. Serra A, Hemu X, Nguyen GKT, Nguyen NTK, Sze SK, Tam JP. A highthroughput peptidomic strategy to decipher the molecular diversity of cyclic cysteine-rich peptides. Sci Rep. 2016;6: Article number: 23005.

59. Serra A, Gallart-Palau X, See-Toh RS, Hemu X, Tam JP, Sze SK. Commercial processed soy-based food product contains glycated and glycoxidated lunasin proteoforms. Sci Rep. 2016:6:26106.

60. Chan C, Thurnherr T, Wang J, Gallart-Palau X, Sze SK, Rozen S, Lee CG. Global re-wiring of p53 transcription regulation by the hepatitis B virus $X$ protein. Mol Oncol. 2016;10(8):1183-95.

61. Community BO: Blender - a 3D modelling and rendering package. Stichting Blender Foundation, Amsterdam Retrieved from http://www.blender.org 2018. Accessed 02 Nov 2020.

\section{Publisher's Note}

Springer Nature remains neutral with regard to jurisdictional claims in published maps and institutional affiliations. 